\title{
Un siècle de diffusion des services aux entreprises dans les systèmes urbains français, britannique et ouest-allemand
}

Cet article a été publié dans une forme révisée dans la revue l'Espace Géographique, 2001, « Un siècle de diffusion des services aux entreprises dans les systèmes urbains français, britannique et ouest-allemand », l'Espace Géographique, 2001 (1), 14p.

\section{A century of producer services ' spatial diffusion : the case of Great-Britain, West-}

\section{Germany and France}

Sophie Baudet-Michel, Université de Paris VII, UMR Géographie-Cités.

Sophie.baudet-michel@univ-paris-diderot.fr

Mots clés : métropole, système urbain, services aux entreprises, diffusion, réticulaire, primatial.

Keywords : High-rank cities, system of cities, producer services, spatial diffusion, network urban system's type, centralised urban system's type.

Résumé : Dans un contexte européen toujours plus prégnant, on compare les comportements des systèmes urbains. L'Allemagne de l'Ouest, la France et la Grande-Bretagne sont retenus, car chacun de ces espaces met en valeur une forme particulière de système de villes : du système réticulé ouest-allemand au système macrocéphale français. Observer l'évolution de la distribution spatiale des services aux entreprises sur le temps long est une manière d'explorer l'influence de la forme des systèmes de villes sur les modalités de la diffusion spatiale des innovations. Trois phases principales sont repérées, qui mettent en valeur des comportements différents, fonction de la forme de la hiérarchie urbaine. Ainsi si le processus de diffusion est rapide lors de l'apparition de l'innovation en Allemagne de l'Ouest, il est ralenti par la suite, alors que c'est l'inverse en France.

Abstract : In an ever more prominent European context, the aim is to compare urban systems' s performances, as systems of cities. West-Germany, Great-Britain and France are chosen, as each of them represent a very specific urban system type : the centralised type is represented with France and the network type with West-Germany. Tracing from 1850 producer service's spatial diffusion, allows to underline three major phases which find their spatial counterparts in an urban perspective. Baring this in mind, national structural differences can be dug out : a great number of high rank cities in a network type of system as that of West-Germany intensifies the diffusion speed in the appearance phase, whereas the spreading is slowed down in the expansion phase ; so much, that at the end of the period, the saturation stage is still to be reached. The opposite occurs in a centralised type of system as that of France.

L'émergence du cycle de l'industrie au milieu du XIXe siècle, a permis à nombre de villes européennes de valoriser fortement leur position. Il semble désormais qu'une partie du dynamisme économique des villes repose sur la fabrication d'information (Castells, 1989). Et la question se pose donc des villes qui pourront ou sauront en tirer bénéfice pour leur croissance. Pour l'heure, l'avantage est de l'avis de tous, accordé aux très grandes villes. Mais cet avantage persistant du niveau métropolitain renvoie d'abord à l'étape actuelle de diffusion de l'économie de l'information, et des activités qu'elle encourage. On sait en effet que les innovations apparaissent d'abord dans les grandes villes, dans les métropoles, pour diffuser par la suite à l'ensemble des systèmes urbains. Or l'économie de l'information peut encore être considérée comme une innovation, étant donné qu'elle se développe surtout à partir des 
années 1970. Le développement des services aux entreprises relève de cette économie de l'information. Leur concentration, que l'on interprète souvent comme symptomatique d'un processus prégnant de métropolisation des systèmes de peuplement, de concentration des activités de direction dans un petit nombre de villes globales (Veltz, 1996, Sassen, 1991) adopte des formes différentes selon l'organisation hiérarchique des systèmes urbains. Juillard et Nonn (1976), Moriconi (1994), on montré que l'on peut identifier deux formes extrêmes des réseaux de villes: une organisation macrocéphale, centralisée et une organisation pluricéphale, réticulaire, entre lesquelles se rangent un continuum de situation intermédiaire. Sachant cela, ce travail cherche à comprendre comment l'économie de l'information modifie l'espace des réseaux urbains nationaux selon leur forme hiérarchique. En effet, dans une perspective dynamique, les changements que les préférences de localisation de l'économie de l'information mettent en valeur, à savoir les préférences métropolitaines, soulèvent la question de l'évolution des réseaux urbains nationaux : quelle est l'ampleur de ces changements géographiques, entraînent-ils des modifications dans l'organisation hiérarchique nationale des systèmes urbains tels que le niveau des grandes villes se démarque encore davantage de l'ensemble ? Et si oui, cela va-t-il de pair avec l'émergence d'un système urbain européen qui pour l'instant concerne essentiellement les villes de plus de 200000 habitants (Cattan et alii, 1994)?

Une démarche comparative permet de répondre en partie à ces questions, en dégageant ce qui d'une part relève des effets conjoncturels de la diffusion des cycles d'innovations économiques, de ce qui exprime les effets structurels de la forme des réseaux de villes. L'étude résumée ici compare le processus de diffusion des services aux entreprises dans les systèmes urbains français, anglais et ouest-allemand entre 1850 et 1990 . On rappelle d'abord quelques unes des règles élémentaires de la théorie de la diffusion spatiale des innovations sur lesquelles on s'est appuyé pour comprendre l'évolution de la répartition spatiale des services aux entreprises. Puis on explicitera les hypothèses de base et enfin on exposera et discutera les résultats obtenus.

\section{Diffusion d'une innovation économique dans les systèmes urbains}

Un processus de diffusion spatiale est un phénomène de propagation dans le temps et dans l'espace d'une chose spécifique (objet, institution, idée, pratique, etc.) auprès des individus, des groupes, ou de toutes autres unités potentielles. Définir ce processus revient à déterminer selon quelles modalités, à quel rythme et sous quelle forme un phénomène s'est progressivement propagé et inséré dans un espace donné, (Saint-Julien, 1982). Le processus de propagation d'une nouveauté dans un système de villes met en effet en jeu divers acteurs : l'innovation, ici les services aux entreprises, les adoptants potentiels, ici les villes.

\subsection{Services aux entreprises, une innovation de l'économie de l'information}

L'économie de l'information marque la montée en puissance des technologies, des processus, des techniques liées au traitement de l'information (Castells, 1989). Elle répond à la mondialisation de l'économie et des marchés. Le rôle de l'information est en effet grandissant pour la gestion des grands complexes multinationaux (Marshall, 1988), pour la diversification des produits et services, pour les changements dans l'utilisation des technologies. Ces modifications entraînent le développement de sociétés de services aux entreprises, entendus comme des aides à la gestion, à la décision, à la prospection, à l'évaluation pour les sociétés de services ou de production. 
Il n'existe pas de consensus clair sur les limites à attribuer au champ d'action des activités de services aux entreprises. Marshall les définit comme les services dévoués aux entreprises et organisations gouvernementales plutôt qu'aux individus, qu'ils opèrent dans des secteurs aussi différents que l'agriculture, les mines, l'industrie et les services (Marshall, 1988).

L'hétérogénéité des activités de services aux entreprises, illustrée par le regroupement dans cette catégorie de services, d'activités à haute valeur ajoutée (ou requérant des personnels aux niveaux de qualifications élevés tels que conseillers juridiques, financiers, sociétés d'ingénierie) ainsi que d'activités à faible valeur ajoutée (au personnel peu qualifié tels que les sociétés de nettoyage, de gardiennage, de travail temporaire) ne fait qu'ajouter à la complexité du problème. Certains auteurs différencient donc activités de services aux entreprises de type tertiaire supérieur (parfois appelées activité basée sur l'information et la connaissance (knowledge-information based, ou activités quaternaires) et activités simples de services aux entreprises. Beyers (1991) les définit comme un ensemble d'activité allant de la finance, l'assurance, l'immobilier, les services légaux et professionnels aux administrations centrales de tous les types d'organisation économiques. Toujours dans cette perspective trans-sectorielle Bailly et Maillat (1991) identifient quatre types majeurs de fonctions, la production, la circulation, la distribution et la régulation. Dans ce cas les services aux entreprises appartiendraient essentiellement aux deuxième et quatrième groupes. Monnoyer, Leo et Philippe ( $1991^{\circ}$ reprennent à leur compte une différenciation entre activités quaternaires et activités tertiaires, différentiation issue de l'économie urbaine transactionnelle.

Si toutes les études s'accordent pour souligner le fait que ces services adoptent d'abord des localisations métropolitaines, en raison à la fois de la proximité des marchés (sièges sociaux) et d'une main d'œuvre très qualifiée, l'hétérogénéité du groupe des services aux entreprises se traduit aussi par des dynamiques spatiales différenciées. Daniels montre ainsi que les sociétés de comptabilité au Royaume-Uni se sont déployées dernièrement pour coloniser les plus grandes villes de provinces (Daniels, 1991). Djellal observe un déploiement semblable des sociétés de conseil en information en France (Djellal et Moulaert, 1995). D’après Marshall, les services à l'industrie les plus ordinaires seraient localisés près des usines et c'est la capitale régionale qui fournirait les services aux entreprises plus spécifiques et rares. Jouvaud (1996) montre que l'intensité des relations avec le marché, l'accessibilité, les contraintes de recrutement jouent sur les préférences de localisation de différentes catégories de services aux entreprises.

Gardant à l'esprit qu'il s'agit à la fois d'éclairer le fonctionnement particulier de chacun des systèmes urbains observés par l'examen des processus de diffusion de l'innovation économique, et de cerner l'ampleur du processus de métropolisation (pris ici comme la concentration grandissante des activités de haut niveau dans les grandes villes) dans chacun d'eux, il nous semble pertinent de retenir essentiellement les catégories les plus représentatives l'économie de l'information (Castells, 1989), celles qui sont les plus porteuses de valeur ajoutée. C'est pourquoi nous avons décidé de nous limiter à la publicité, aux services de marketing, aux activités de recherche et développement, aux services techniques et d'ingénierie, aux organisations économique et professionnelles, ainsi qu'aux services d'informatique.

\subsection{Les annuaires Kompass, source d'information}

Deux problèmes sont à résoudre d'emblée : celui de la comparabilité des nomenclatures qui dans les trois pays définissent les services aux entreprises ; celui de l'objet géographique dans 
lequel ces services sont localisés. Les sources d'informations qui se fondent sur des classifications d'activités économiques homogènes pour tous les pays européens (nomenclature NACE pour la CEE), recensent l'emploi et ne sont disponibles que pour des dates très récentes, à un niveau d'agrégation élevé, la plupart du temps régional (notamment EUROSTAT). Dès lors il est apparu qu'il serait nécessaire de constituer une base de données reposant sur des sources privées, et donc non exhaustives, ici les annuaires Kompass.

Les entreprises recensées en 1994 par Kompass dans chacun des trois pays sont les plus importantes au regard de leur chiffre d'affaire, avec un souci de description de l'ensemble des secteurs économiques. On y trouve des entreprises aux activités de tout type, de toutes tailles (en nombre d'emploi), et leur localisation est précisée. La même méthode de collecte de l'information est appliquée dans les trois pays. Ces entreprises, si elles ne constituent qu'un infime pourcentage ( 0,1 environ) du nombre total des sociétés, participent très largement à l'activité économique des pays (plus de $80 \%$ de l'activité financière et $70 \%$ de la force de travail). Pour chacune d'entre elles on dispose d'informations relatives à leur taille tant en terme de masse salariale que de chiffre d'affaire, ou même de chiffre d'affaire à l'exportation. Néanmoins ces informations, beaucoup moins persistantes sur la durée que la simple existence des entreprises n'ont pas été utilisées.

En Allemagne de l'Ouest en 1994, 4026 sociétés recensées par Kompass agissent dans le secteur des services aux entreprises (les services de publicité, de marketing, les activités de recherche et développement ou d'ingénierie, les organisations économique et professionnelles, ainsi que les services d'informatique), et sont localisées dans les agglomérations de plus de 100000 habitants. En France, 10819 sociétés sont dans ce cas pour 3000 en Grande-Bretagne ; ces différences sont le reflet de l'inégale ampleur des enquêtes faites par Kompass dans chaque pays, puisqu'en France 115000 entreprises sont renseignées, 50000 en Allemagne de l'Ouest et 25000 en Grande-Bretagne. Cependant, malgré l'inégalité du volume des échantillons en 1994, il faut souligner que, pour la France et l'Allemagne de l'Ouest, le nombre d'entreprises en 1850 est le même. On a multiplié les indicateurs utilisés pour l'analyse de la diffusion spatiale : certains annulent en partie l'effet de taille des échantillons d'entreprises (pyramide des âges des entreprises, âge médian et moyen des entreprises), d'autres (utilisation de différents seuils d'apparition des entreprises) permettent de tester la robustesse des résultats (Michel, 1998).

Les établissements recensés sont principalement des sièges sociaux (86\% pour la France en, 96\% pour l'Allemagne de l'Ouest et la Grande-Bretagne, en 1994).

La France comporte davantage de petites entreprises, mais ses grandes unités sont plus vastes que les plus grandes entreprises allemandes, si bien que la structure française est plus contrastée. Cependant, dans les deux cas, la majorité des sociétés se situe entre un et 100 salariés (85\% en France, 79\% en Allemagne de l'Ouest). Ces résultats sont corroborées par d'autres sources : Woronoff indique que pour les services aux entreprises comme pour l'industrie (Woronoff, 1994), la France se caractérise par un plus grand nombre de sociétés de petites tailles que l'Allemagne de l'Ouest, avec quelques cas exceptionnels d'entreprises surdimensionnées par rapport au reste.

Quant à la répartition des établissements entre les catégories d'activités retenues, elle est assez différente dans les trois pays. Si en France, 50\% des établissements sont répertoriés dans les services informatiques, de conseil en gestion, c'est le cas de $17 \%$ des établissements d'Allemagne de l'Ouest et 33\% de Grande-Bretagne. En Allemagne de l'Ouest, les services 
techniques et d'ingénierie se taillent la part du lion (40\% de l'échantillon) contre $20 \%$ en France et $30 \%$ en Grande-Bretagne, suivis de près par les services commerciaux et de publicité (33\%) contre 19\% en France et Grande-Bretagne. La structure par activité diffère donc sensiblement d'un espace à l'autre, reflétant en cela la structuration générale de l'économie, et la Grande-Bretagne est à mi-chemin entre la France et l'Allemagne de l'Ouest de ce point de vue.

On a insisté précédemment sur l'intérêt spécifique qu'il y a à utiliser ici les annuaires nationaux Kompass ; il n'en reste pas moins que ce type d'information a été jusqu'alors peu utilisé, et que les limites en sont peu connues. Dès lors, il est nécessaire de tenter de mieux définir la nature propre de ces échantillons et d'en évaluer avec plus de précision la validité géographique, notamment en situant ces échantillons dans le cadre plus général d'enquêtes ou de recensements officiels. En ce qui concerne la Grande-Bretagne, on peut confronter les informations obtenues à partir des annuaires Kompass, à celles qui sont fournies au niveau des Travel-To-Work Areas (plus étendues que les agglomérations urbaines) par le recensement de l'emploi, depuis 1971. Pour la France, on peut comparer la base construite à partir des données Kompass, aux données sur l'emploi par activité des recensements de la population française. Les recensements de population au lieu de domicile depuis 1960 permettent de compter les employés des services aux entreprises (selon la nomenclature des produits 1975) à toutes les dates de recensement depuis 1960 dans les 59 unités urbaines de plus de 100000 habitants. On met ainsi en relation la répartition de l'emploi des services aux entreprises dans les villes pour les deux sources aux différentes dates. Les informations censitaires dont on dispose pour l'Allemagne de l'Ouest ne permettent en revanche aucun test.

Dans le cas britannique, comme dans le cas français, les chiffres bruts obtenus par les deux sources sont différents, de par leur nature même, mais les ordres de grandeur de la distribution spatiale des services aux entreprises à partir des chiffres des recensements et de Kompass restent semblables sur toute la période. Les échantillons Kompass ne privilégient pas plus les grandes villes ou les régions méridionales que ne le font les sources exhaustives des recensements en Grande-Bretagne et en France...

\subsection{Une observation sur le temps long : 1850-1994}

L'intérêt de la base Kompass est donc à la fois de permettre une comparaison entre différents pays, sur la base d'une nomenclature d'activité commune, mais aussi de permettre une observation sur le temps long. En effet, les premières créations de sociétés de ce type (un peu plus d'une centaine) sont datées de la décennie 1850. Leur ancienneté atteste non pas de l'existence des services aux entreprises, mais des germes qu'elles portaient alors d'une future maturation des activités de services aux entreprises : les travaux de Landes (1975) sur l'économie européenne montrent en effet que la deuxième moitié du XIXe enregistre en Europe l'essor d'activités juridiques, financières, d'assurances, de réclame, de diffusion des informations (expansion des journaux) qui portent en elle les germes de l'économie de l'information. L'innovation que ces activités représentent est en effet prise ici comme le résultat d'un processus graduel de changement, dans un contexte spécifique (Amendola et Gaffard, 1988). De surcroît, suivre le processus de diffusion de ces services depuis la deuxième moitié du XIXe siècle s'avère utile pour prendre en compte la temporalité longue des dynamiques urbaines.

Nous avons choisi de travailler à partir d'une seule source, l'annuaire 1994, qui permet de connaître pour chacune des entreprises mentionnées sa date de création. Ainsi le nombre 
d'entreprises dénombrées n'est pas affecté par les progrès et les fluctuations d'enregistrement des entreprises par Kompass. Connaissant la date de création, et la localisation des sociétés de services aux entreprises recensées par les annuaires, on suit l'émergence de cette activité. La base développée recense les entreprises en 1994, certaines ayant été créées dans les années 1850. La durée d'observation a été segmentée en période de 10 ans, en affectant par addition à chacune des décennies successives le nombre d'établissements créés au cours de la période correspondante, ce depuis 1850.

Les deux inconvénients majeurs de cette méthode sont d'une part d'ignorer les entreprises qui ont été créées puis ont disparu avant 1994, d'autre part de gonfler les chiffres de la dernière décennie par rapport aux périodes antérieures. En effet, l'ensemble d'entreprises sur lequel on travaille est un échantillon de survivants. Il est donc inévitable qu'il soit d'autant plus élevé que la période examinée est récente. Toutefois on sait que les taux de mortalité et de natalité des jeunes entreprises sont très élevés et qu'au delà de dix années, l'âge de l'entreprise ne peut plus être mis en relation avec sa capacité à survivre (Keeble et Walker, 1994). On peut donc supposer que les taux de croissances du nombre d'entreprises de l'échantillon à chaque décennie (exceptée la dernière) sont un reflet assez fidèle des taux de croissance réels de cette activité.

\subsection{L'espace d'étude}

France, Allemagne de l'Ouest et Royaume-Uni ont été retenus car ces trois pays présentent un degré d'urbanisation similaire, ont enregistré les mêmes bouleversement historiques et ont suivi des trajectoires économiques semblables au cours des cent cinquante dernières années : le niveau de vie y est élevé, le système de production orienté vers le marché, ils sont à peu près au même stade de développement (Rostow 1978). Leurs réseaux de villes peuvent être appréhendés comme des systèmes, c'est à dire " ensemble national de villes interdépendantes dans le sens ou chaque changement significatif dans l'activité économique, la structure d'emploi, le revenu, ou la population de l'une des villes, élément du système, produira directement ou indirectement quelque modification dans l'activité économique d'une ou plusieurs autres villes du système" (Pred, 1975). L'innovation diffuse dans un système ayant une structure, définie comme l'arrangement des adoptants potentiels, les villes.

Le choix de ces systèmes urbains répond aussi à l'identification des deux types principaux d'organisations des systèmes de villes, centralisés et réticulés. L'espace national français est caractéristique du type de réseau urbain centralisé ou parisien. On a donc retenu le territoire métropolitain français dans ses limites actuelles. L'ancienne république fédérale d'Allemagne offre en revanche un système urbain de type réticulaire. Si, sur le plan conceptuel, le fait de ne pas tenir compte des modifications d'extensions territoriales qu'ont connues l'Allemagne et la France pose problème, cette attitude se justifie dans le cadre et la problématique choisis ici. En effet c'est à partir des années 1950 que les services aux entreprises prennent véritablement leur essor (Greenfield, Marshall, Beyers, Daniels), et c'est à partir de cette date qu'il faut examiner les systèmes urbains dans des limites géographiques cohérentes. Or l'évolution de l'Allemagne de l'Ouest et de son réseau urbain s'est faite de manière tout à fait indépendante de l'Allemagne de l'Est depuis 1950, et ce jusqu'en 1989. D'autre part, si notre étude se prolonge jusqu'en 1994, on peut considérer que les retentissements de la réunion des deux Allemagne sur le réseau urbain seront peu visibles au début des années 1990. Enfin, si l'on a pris en compte une période très antérieure (1850-1950), au cours de laquelle le territoire allemand a été plusieurs fois et très largement remanié, la croissance des services aux entreprises sur cette période concerne un petit ensemble de villes du réseau ouest-allemand. 
Le territoire de la Grande-Bretagne est un bon témoin de ce que serait un type urbain intermédiaire.

Ayant choisi d'observer les systèmes urbains à l'échelle nationale, et dans une perspective diachronique, la démarche s'est trouvée immédiatement confrontée à la question de l'hétérogénéité de la définition des villes selon les pays. Les travaux menés par MoriconiEbrard (Géopolis) permettent de s'appuyer sur une définition homogène dans l'espace et le temps, celle des agglomérations urbaines. Le choix de cette définition se justifie encore à l'heure actuelle par la concentration des établissements de services aux entreprises dans la zone centrale des bassins d'emploi. Néanmoins, notons que sur la totalité des sociétés de services aux entreprises recensées sur l'ensemble du territoire national de chacun des pays, en France $80 \%$ sont localisées dans l'espace des agglomérations de plus de 100000 habitants, en Allemagne de l'Ouest $61 \%$ et plus que 55\% en Grande-Bretagne. Cette inégale représentation des services aux entreprises sur le territoire des agglomérations de plus de 100000 habitants est plus à porter à l'actif de la diversité des formes fonctionnelles de la ville dans les trois pays, qu'à celui d'une inégale diffusion interurbaine des services aux entreprises. L'inégale "représentativité" des échantillons de sociétés de services aux entreprises selon les pays enregistre ici les limites inhérentes à l'utilisation d'une définition uniforme de la ville pour les trois pays, sans laquelle la comparaison ne serait pourtant pas possible.

Il ressort de nombreuses études (Pumain, Saint-Julien, 1994, Le Filattre, 1964, Smailes, 1971, Bourne, 1978) que c'est un seuil de taille d'environ 100000 habitants qui différencie les villes du niveau supérieur de la hiérarchie urbaine du reste des villes d'un système urbain national, tandis que le niveau métropolitain s'exprimerait au niveau des villes de 500000 voire d'un million d'habitants. C'est pourquoi le niveau des agglomérations de plus de 100000 habitants est apparu pertinent pour appréhender la dynamique du cycle de l'information dans les systèmes de villes, ce qui représente pour chaque pays un échantillon d'une soixantaine d'agglomérations urbaines.

La dimension spatiale de la base a été obtenue à partir des données fournies par Kompass, et grâce aux informations contenues dans Géopolis. L'annuaire Kompass indique l'adresse communale de chaque établissement. La base Géopolis définit la composition "communale" (Gemeinden pour l'Allemagne, communes pour la France, « villages » pour la GrandeBretagne) de chacune des agglomérations. On a donc attribué à chaque agglomération urbaine les établissements localisés dans les communes appartenant à cette même agglomération. $\mathrm{Ne}$ connaissant la localisation des établissements que pour l'année 1994, il a été décidé d'attribuer cette localisation à toute la période étudiée : les délocalisations et relocalisations éventuelles ne peuvent donc être suivies. Mais on considère cela comme une perte d'information mineure, les services aux entreprises n'ayant pas subi l'équivalent des énormes phases de restructurations qui ont affecté nombre d'entreprises et d'établissements du secteur secondaire. Les études d'évolution des localisations des services aux entreprises montrent en effet à ce jour que les relocalisations sont rares, et qu'elles se font dans le périmètre même des villes dans lesquelles ces services sont implantés, du centre de ces villes vers leur périphérie (Sassen (1991), Noyelle et Stanback (1991)).

Observant dans trois systèmes urbains aux formes différentes, la diffusion des services aux entreprises on constatera la spécificité de leur logique de localisations. En effet, chaque cycle économique met en exergue l'opérabilité de certaines échelles d'espaces, et partant, de certaines situations tant hiérarchiques que géographiques des villes (Ascher, 1995, Pumain, 1994). On abordera ensuite la question de l'influence de la structure hiérarchique du réseau urbain sur le fonctionnement de l'accroissement et de la diffusion de cette activité. 


\section{Métropolisation et cycle de l'information : des expressions spatiales fonctions de la}

\section{forme des systèmes urbains}

La première conclusion de cette étude reprend l'observation (Beyers, 1991, Daniels, 1985, Illeris, 1991) selon laquelle l'économie de l'information, et les activités qui lui sont liées, souligne le niveau métropolitain des systèmes urbains. Plusieurs éléments viennent conforter ce résultat fondé sur l'extraordinaire concentration des sociétés de services aux entreprises au niveau des villes millionnaires en général. Ces agglomérations bénéficient en effet d'avantages comparés importants de par leur forte accessibilité, par la présence de personnel qualifié, par l'accumulation d'information qui s'y manifeste...

\subsection{Partout, le niveau métropolitain}

La notion de métropole est l'objet de différentes définitions. Si l'on s'accorde généralement pour dire que ce sont des villes de grandes tailles (au moins millionaires), certains chercheurs les définissent comme des villes interface entre un système de villes internationales et les systèmes nationaux de villes. Le niveau des métropoles est alors identifiable grâce à de la césure maximale que l'on observe dans une distribution de taille des villes ; cette discontinuité dans la hiérarchie signale les villes qui doivent leur taille à une position d'ouverture internationale et valorisent des points privilégiés de contact entre le système urbain national et le monde (Moriconi-Ebrard, 1994) ainsi souligné, comprend les villes les plus diversifiées de chaque pays. C'est cette définition que l'on privilégie ici.

On a observe ainsi qu'en 1850, les villes métropolitaines (Hamburg, Essen, Dusseldorf, Köln, Francfurt, Berlin, Stutgart et München, Paris et Londres) rassemblent l'essentiel des sociétés de services aux entreprises des échantillons. En 1990, la concentration des services aux entreprises dans les villes métropolitaines demeure.

Il existe donc des effets qui pour l'instant apparaissent comme des effets de barrières perméables au niveau métropolitain, puisque en deçà d'un certain niveau de villes, la présence des services aux entreprises est faible.

Camagni (1992), observant les villes d'Europe, voit l'émergence d'un réseau urbain européen, s'appuyant sur plusieurs types de réseaux de villes, et notamment des réseaux spécialisés dans les activités du tertiaire supérieur ; Cattan et Saint-Julien (1998) identifient elles aussi plusieurs réseaux de villes alimentant la formation d'un système urbain, d'échelle européenne : parmi ceux-ci, outre le réseau des capitales, elles aussi signalent celui des villes spécialisées. Il nous semble ici, que la répartition très concentrée des services aux entreprises montre l'appartenance des métropoles à l'un de ces réseaux spécialisés. Le fait que cette spécialisation 'informationnelle', relevant d'un type d'organisation économique émergent, favorise les nœuds centraux du système urbain européen (Cattan $(1998$; 1992), Rozenblat (1993)), n'est probablement pas indépendant de la montée en puissance d'une échelle de fonctionnement internationale des espaces nationaux européens.

\subsection{Deux formes du niveau métropolitain pour deux formes de concentration métropolitaines des services aux entreprises}

Or chaque pays participe à l'aune de sa propre organisation hiérarchique à cette émergence d'un système européen de villes. Ainsi, si Londres et Paris sont actuellement deux piliers incontestables, voire centraux, de cette organisation urbaine affleurante, l'Allemagne marque davantage sa présence par le nombre de ses métropoles de rang international que par la prégnance d'une ville unique et macrocéphale. Dans une perspective nationale, cette inégale 
participation des hiérarchies urbaines, à travers un seul ou plusieurs point d'interface, peut avoir des répercussions sur le fonctionnement interurbain, et modifier les modes de développement territoriaux existants.

En 1990, les contextes urbains français et britannique engendrent une concentration extraordinaire des services aux entreprises dans la capitale (Paris, $48 \%$ des sociétés pour 38\% de la population ; Londres $37 \%$ des sociétés pour $27 \%$ de la population), alors que le degré de concentration des sociétés de services aux entreprises, répond très exactement à celui de la population en Allemagne de l'Ouest (8 villes, pour $61 \%$ de la population et $61 \%$ des sociétés de services aux entreprises). S'opposent ainsi l'organisation du système ouest-allemand où concentration de la population et concentration des services aux entreprises sont similaires, aux organisations primatiales française et britannique dans lesquelles la concentration des services aux entreprises est supérieure à celle de la population.

Dans le contexte actuel d'union européenne, quelle forme hiérarchique des réseaux urbains paraît la plus adaptée à la diffusion du cycle de l'information ? Examiner le déroulement du processus de diffusion des services aux entreprises dans ces trois réseaux apporte des éléments de réponses à cette question. Mais, dans une Europe en expansion, cet examen conduit aussi à ré-interroger les conditions de la dynamique des systèmes urbains nationaux.

\section{Diffusion des services aux entreprises : des différences entre systèmes urbains}

Le nombre de sociétés de services aux entreprises croît de manière exponentielle dans les trois pays (Tableau 1 ; Figure 3 ) et on peut distinguer trois périodes de développement des échantillons : 1850 à 1950-1960 est une périodes de gestation de l'activité ; l'accroissement des échantillons est très lent, il est le fait de l'apparition d'activités dépendant du développement industriel (publicité mais surtout société d'ingénierie). 1950-1990 est une période de développement plus importante et plus rapide des échantillons. Castells (1989) a montré que les services aux entreprises se sont développés par externalisation et en réponse à la complexification croissante des marchés économiques, à la concurrence accrue entre entreprises, à l'internationalisation de l'ensemble des échanges économiques et donc finalement à un besoin informationnel accru. Pour les dernières années (1990-1194), la diffusion semble entrer dans la période de saturation, à partir de laquelle l'activité diffuse peu dans l'ensemble des villes, le nombre de société de services aux entreprises que l'on observe augmente peu au cours de ces années.

Hägerstrand (1967) distingue trois périodes dans un processus de diffusion : l'apparition de l'innovation qui différencie fortement les lieux entre eux, l'innovation reste concentrée dans un petit nombre de lieux ; la période d'expansion au cours de laquelle l'innovation se propage rapidement, apparaissant dans la plupart des lieux ; enfin la période de condensation qui voit un ralentissement net, le nombre de lieux restant à atteindre étant faible. Les travaux de Rogers (1983) incitent à distinguer deux phases dans la période d'apparition, une phase d'émergence et une phase de diffusion précoce qui permettent de différencier les adoptants innovateurs des adoptants précoces ; selon cette distinction la propagation de l'innovation dans l'espace s'accélère avec la diffusion précoce sans atteindre pour autant la vitesse de la phase d'expansion.

Des indicateurs divers (pyramide des âges, âge médian, âge moyen des entreprises, seuil de 1, 5, 10, 20 entreprises par ville) ont été utilisés pour observer le processus de diffusion des services aux entreprises dans les trois systèmes de villes. On a ainsi calculé l'âge moyen et l'âge médian des sociétés de services aux entreprises d'une ville en 1994 et on a supposé que 
les villes dans lesquelles cet âge est le plus élevé sont celles dans lesquelles est d'abord apparu cette innovation. Pour les pyramides des âges, on a supposé que lorsque la pyramide des âges des sociétés de services aux entreprises d'une ville est de type jeune, c'est que l'innovation y est apparue plus récemment que si cette pyramide est vieillie. Des indicateurs de seuil d'apparition des sociétés de services aux entreprises(date à laquelle on compte 1, cinq, dix, vingt entreprises dans une ville) permettent eux aussi de dater l'ancienneté de l'innovation dans une ville. Tous ces indicateurs mettent en valeur les mêmes phénomènes (Michel, 1998).

On a choisi ici de rapporter les résultats obtenus pour l'observation de la diffusion à partir du seuil de présence de 10 sociétés de services aux entreprises par agglomération car c'est à la fois un indicateur facile à présenter et dont le comportement résume bien l'ensemble des observations. Ainsi on distinguera pour observer la diffusion des services aux entreprises deux phases qui appartiennent à la période d'apparition (phase d'émergence et de diffusion précoce), et deux périodes qui suivent : l'expansion et la saturation (Tableau 2). Les limites de début et de fin des phases sont déterminées à la fois d'après les courbes de fréquences cumulées des villes équipées (Figure 1), les taux de diffusion (Figure 2), les taux d'accroissement des échantillons (Figure 3).

\subsection{L'apparition : diffusion intense et rapide en Allemagne, faible et tardive en France}

Les phases d'émergence et de diffusion précoce s'individualisent nettement de la période de diffusion massive qui suit. Ces deux phases ont été identifiée par des ruptures dans les courbes de croissance du nombre de sociétés de services aux entreprises et du nombre de villes touchées par l'innovation.

\subsubsection{La phase d'émergence : intense en Allemagne de l'Ouest, faible en France}

La croissance de l'activité des services aux entreprises au cours de la phase d'émergence est lente (Figure 3) et en France, à la fin de la phase d'émergence (1850-1930), six villes sont touchées par l'innovation (Carte 1, Tableau 2). Il s'agit des quatre plus grandes villes françaises de l'époque (Lille (33 sociétés), Lyon (34), Marseille (23), Paris (222)), auxquelles viennent s'ajouter Strasbourg (16) et Toulouse (14). Mais les services aux entreprises sont restés exclusivement concentrés dans l'agglomération parisienne, jusqu'au début du siècle, et en 1930 encore une très forte césure sépare Paris de Lyon! Ainsi, le processus de diffusion a fonctionné, mais une barrière quasi absorbante sépare Paris du reste du système urbain. Au cours de cette première phase, l'essaimage des services aux entreprises est lent, la croissance de l'activité reste concentrée dans la capitale, inhibant la diffusion interurbaine de l'activité.

En Allemagne de l'Ouest, à la fin de cette phase d'émergence (1850-1930), le nombre de villes "contaminées" est beaucoup plus important (Tableau 2, Carte 1), et les sociétés de services aux entreprises sont réparties de manière peu discriminante dans près d'une quinzaine de villes (Berlin (42), Bielefeld (13), Bremen (24), Düsseldorf (50), Essen (58), Frankfurt (47), Hamburg (53), Hannover (21), Köln-Bonn (45), Mannheim (12), München (37), Nürnberg (18), et Stuttgart (36)) : dès 1880, les huit villes qui constituent le sommet actuel de l'armature urbaine allemande accueillent toutes au moins une dizaine de sociétés de services aux entreprises.

\subsubsection{La phase de diffusion précoce : longue en Allemagne de l'Ouest, courte en France}


En France, la phase de diffusion précoce a lieu des années 1930 aux années 1950 et deux villes (Bordeaux et Nancy) de plus s'équipent. Les changements dans la distribution interurbaine des services aux entreprises sont faibles, la croissance de l'activité se porte essentiellement sur les villes déjà touchées. Ainsi, les différenciations de la période précédente se perpétuent largement, et l'organisation primatiale du niveau supérieur de la hiérarchie urbaine est toujours très sensible dans les inégalités de la distribution interurbaine des services aux entreprises (Tableau 2, Carte 2).

En Allemagne de l'Ouest, la phase de diffusion précoce dure plus longtemps (40 ans au lieu de 20). La diffusion des services aux entreprises se prolonge pour atteindre près d'une trentaine de villes (28), mais elle ne masque pas un effet de seuil entre les huit plus grandes villes et le reste du réseau urbain (Tableau 2, Carte 2), qui se manifeste par la perpétuation de la concentration des services aux entreprises ( $60 \%$ des sociétés) dans ces villes à toutes les périodes.

En Grande-Bretagne, la période d'apparition des services aux entreprises va de 1910 aux années 1960. A cette date, 13 villes accueillent au moins cinq sociétés de services aux entreprises chacune (le seuil est inférieur en raison de la faiblesse de l'échantillon sur toute la période), 10 en hébergent plus du double (Tableau 2, Carte 2). Ces villes rassemblent souvent plus de 500000 habitants ( Londres (126), West-Midlands-Birmingham (30), Manchester (15), Bristol (15), Glasgow (13), Sheffield (14), Newcastle (10), West-Yorkshire-Leeds (11)), auxquelles viennent s'ajouter Coventry (5), Cambridge (6), Derby (10), Leicester (11). En Grande-Bretagne, la diffusion fonctionne rapidement et un grand nombre de villes est équipé à la fin de la période d'apparition. En cela la Grande-Bretagne ressemble davantage à l'Allemagne de l'Ouest qu'à la France. Mais à l'instar de Paris, Londres rassemble quatre fois plus de sociétés de services aux entreprises que la seconde ville à l'époque, c'est à dire Birmingham.

Les décalages des taux de croissance des échantillons reflètent les décalages dans les processus d'industrialisation et de tertiairisation nationaux tandis que le taux de diffusion (Figure 2) nettement individualisée par pays, signale le rôle des systèmes urbains dans la diffusion. On peut donc penser que les avances et les retards dans la croissance du nombre de villes (taux de diffusion) dans lesquelles on trouve les sociétés de services aux entreprises révèle une part du fonctionnement dynamique des hiérarchies urbaines.

En Allemagne de l'Ouest, où le niveau métropolitain est pluricéphale, la diffusion est intense et rapide au départ (les taux de diffusion sont plus élevés que les taux de croissance), particulièrement en début de période. Si bien que l'ensemble des agglomérations du niveau supérieur de la hiérarchie est vite équipé (dès 1880), permettant que la totalité du territoire ouest-allemand soit desservie par plusieurs points forts, éloignés les uns des autres. En France, dans un réseau où la primatie est très forte, la diffusion est très faible lors de l'apparition de l'innovation. L'accroissement du nombre de sociétés de services aux entreprises est très lent, celui du nombre de villes qui accueille ces sociétés l'est encore plus et Paris conserve longtemps (plus de 30 ans) le monopole des services aux entreprises. La diffusion interurbaine ne fonctionne pas, l'effet de barrière entre le niveau métropolitain et le reste du système urbain apparaît d'autant plus fort qu'il est le fait d'une seule grande ville. En Grande-Bretagne, dans un système où la primatie est plus atténuée, où un niveau de grandes villes au pouvoir fort existe, l'accroissement du nombre de sociétés de services aux entreprises est moyen, celui du nombre des villes qui accueillent ces sociétés aussi. La diffusion lors de la période d'apparition (émergence + diffusion précoce) fonctionne à 
l'intérieur du niveau métropolitain, l'effet de barrière entre la ville primatiale et les autres villes est nettement atténué.

\subsection{L'expansion : diffusion massive et rapide en France, diffusion lente en Allemagne de l'Ouest}

La période d'expansion, correspond, dans un processus de diffusion urbaine, à l'augmentation forte du nombre de villes touchées par l'innovation. Les trois systèmes obéissent à cette règle générale, avec cependant des différenciations nettes (Tableau 2). Cette période se caractérise normalement par son intensité et donc par le nombre important de lieux touchés, c'est une étape de rattrapage des plus forts contrastes entre les villes. En France, elle se déroule des années 1950 à 1990, en Allemagne de l'Ouest de 1970 à 1990, en Grande-Bretagne à partir des années 1960. Ces dates de début et de fin de phase sont identifiées sur les courbes de croissance du nombre de sociétés et du nombre de villes touchées.

En France, au cours de cette période un très grand nombre de villes est touché (Carte 3), le rattrapage est massif, sans que pour autant les inégalités de la distribution des services aux entreprises ne s'estompent vraiment. Ainsi, en 1990, seules deux villes restent à l'écart. Cette diffusion massive, qui est notamment le fruit d'un accroissement très fort du nombre d'entreprises de l'échantillon est d'autant plus remarquable que l'effet de barrière que constitue la primatie parisienne a malgré tout très largement persisté, et qu'en 1990 la concentration des services aux entreprises reste supérieure à celle de la population dans la capitale.

En Allemagne de l'Ouest la période d'expansion se traduit par un fort essaimage des services aux entreprises, mais la progression du nombre de villes touchées est moins forte qu'en France (Carte 3). En 1990, une dizaine de villes est encore à l'écart.

En Grande-Bretagne, la progression du nombre de villes touchées s'apparente à celle de l'Allemagne de l'Ouest (Carte3). En 1994, les services aux entreprises sont présents dans 38 villes, mais absents d'une vingtaine d'autres. Comme en Allemagne de l'Ouest, la présence d'un niveau assez fourni de grandes villes semble être un frein à une diffusion aussi massive qu'en France.

Ainsi, on aboutit en fin de période à ce paradoxe qui est que, dans le système urbain français, "théâtre" d'une discontinuité majeure entre la capitale nationale et les capitales régionales, mais dans lequel le niveau des villes moyennes est très fourni, le processus de diffusion atteint finalement un nombre plus important de villes que dans le système urbain ouest-allemand, pluricéphale. Ce résultat est lié à l'accélération de la croissance du nombre de sociétés de services aux entreprises à partir de 1950. Cette accélération intervient au moment où le groupe des villes moyennes, très nombreuses en France commence à s'équiper.

\subsection{Le début de la saturation : 1990-1994}

A partir de 1990, l'accroissement des échantillons est faible (Tableau 1, Figure 3), leur diffusion spatiale aussi (Figure 1 et 2, Tableau 2) et l'on peut penser que ces quatre dernières années de l'étude marque le début d'une phase de saturation.

La phase de saturation semble entamée en France et en Allemagne de l'Ouest dès 1990, date à partir de laquelle la diffusion progresse essentiellement par accroissement dans les zones déjà touchées. De 1990 à 1994 le nombre de villes dans lesquelles on trouve au moins 10 sociétés 
de services aux entreprises ne progresse plus. En Grande-Bretagne, la saturation n'est pas atteinte.

En résumé, les phases d'émergence et de diffusion précoce des services aux entreprises, sont intenses (elles touchent un grand nombre de villes) dans les systèmes urbains ouest-allemand et anglais. En France, c'est lors de l'étape d'expansion que la diffusion spatiale est intense. En 1994, seules deux villes restent encore à l'écart. En revanche, en Allemagne de l'Ouest et en Grande-Bretagne, la période d'expansion est nettement moins intense, le nombre de villes dans lesquelles se développent les services aux entreprises beaucoup plus faible ; en 1994, un nombre important de villes reste à l'écart. On peut donc dire que le démarrage du processus de diffusion interurbaine est plus tardif et plus brutal en France qu'en Allemagne de l'Ouest.

\section{4. Des décalages dans le processus de diffusion induits par la forme des réseaux ?}

Plusieurs principes d'explication peuvent être invoqués pour rendre compte des décalages et des différences d'intensité dans le déroulement du processus de diffusion. Si une explication de nature économique, et qui fait appel au développement industriel décalé dans le temps des trois pays, doit être évoquée, elle n'est pas la seule.

Dans des contextes économiques qui enregistrent les mêmes vagues de croissance, l'accroissement différenciés des échantillons et du nombre de villes touchées souligne des divergences nationales. Si le rythme de la diffusion est plus rapide en Allemagne de l'Ouest lors de la période d'apparition de l'activité, c'est d'abord parce que l'accroissement de l'échantillon de sociétés est rapide au cours de cette période, en réponse à une industrialisation très intense et dispersée. En France, dans le même temps, le processus d'industrialisation, plus lent, mais aussi plus avancé en début de période, est beaucoup plus concentré dans l'espace, et ne concerne qu'un petit nombre de villes. Après la seconde guerre mondiale, pendant les Trente glorieuses, qui bénéficient aux trois pays, le fonctionnement de la diffusion est rapide en France, lent en Allemagne de l'Ouest et en Grande-Bretagne. L'accroissement rapide de l'échantillon et de la diffusion de ce type de services en Allemagne de l'Ouest lors de la phase d'apparition s'oppose à leur diffusion lente par la suite, alors même que le marché des services aux entreprises se diversifie après la seconde guerre mondiale et que la croissance économique s'y maintient fortement.

Dans un processus de diffusion spatiale, l'organisation de l'espace d'accueil de l'innovation intervient à la fois à travers les canaux de la diffusion et les barrières qui occupent l'espace. Dans ce cadre, la structure du système de peuplement est essentielle et l'examen de l'organisation hiérarchique de ce système souligne des barrières à la diffusion. La proximité de deux lieux dépend non seulement de la distance géographique qui les sépare mais aussi de leur position hiérarchique et fonctionnelle (Saint-Julien, 1995).

La question que l'on pose maintenant est donc : Comment les formes de hiérarchie urbaine différencient le processus de diffusion spatiale des services aux entreprises ? Avant de tenter d'y répondre, il est important de présenter plus avant les formes hiérarchiques des trois systèmes urbains.

\subsubsection{Forme hiérarchiques des systèmes urbains}

En s'appuyant sur des travaux précédents (Juillard et Nonn, 1976 ; Moriconi-Ebrard, 1994), nous rappelons qu'il existe trois types de systèmes urbains. Juillard et Nonn ont identifié deux formes d'organisation au niveau régional, le type rhénan, et le type parisien qui peut être 
subdivisé en deux. Moriconi-Ebrard (1993), étudiant les formes de distribution des tailles de villes dans le monde, montre que l'on observe quatre formes principales des systèmes urbains, en fonction des déformations observées par rapport au modèle paretien.

Néanmoins, on pourra, sans trahir cette interprétation élargir ici la notion de métropole, dans deux des trois pays ici considérés (la Grande-Bretagne et la France), à un certain nombre de villes, qui si elles se situent en deçà de la plus grande discontinuité de la hiérarchie de taille des villes, n'en constituent pas moins les points forts des réseaux urbains nationaux, voire d'un réseau européen émergeant. Ces villes rassemblent généralement plus de 500000 habitants.

En France, une discontinuité majeure affecte la hiérarchie concavo-convexe du système urbain français : c'est entre Paris et la deuxième ville française (actuellement Lyon) qu'on peut observer une barrière hiérarchique majeure ; les très grandes villes sont peu représentées, elles sont rejetées en une couronne périphérique alors que le territoire est fort équipé en villes moyennes (de 100 à 500000 habitants). Dans ce système primatial, centralisé, les unités administratives sont nombreuses et grossissent le nombre de villes de taille moyenne. Les liaisons entre ces grandes villes de la périphérie sont très faibles et si certaines d'entre elles (Lille, Lyon, Marseille, Strasbourg et Toulouse) ont rang de métropole, bien peu sont réellement de dimension internationale. Une discontinuité majeure en France est donc située au niveau le plus élevé du système urbain, elle isole Paris du reste du système.

L'Allemagne de l'Ouest, avec huit métropoles, constitue à l'inverse un cas extraordinaire de polycéphalie pour un système urbain convexo-concave. Huit métropoles européennes se sont développées dans le cadre d'un régime économique libéral (Hamburg, Berlin, Essen, Düsseldorf, Köln, Frankfurt, Stuttgart et München). Nurnberg, Mannheim, Bielefeld, Hannover, Bremen et Karlsruhe viennent enrichir à un niveau immédiatement inférieur cette classe de très grandes villes et de ce fait, le niveau suivant, des villes moyennes ( de 100 à 500 000 habitants) est plus faiblement représenté. Ce développement accentué des très grandes villes, sans qu'aucune ne domine l'ensemble du réseau urbain, est avant tout la traduction du fonctionnement fédéral du territoire national, dans lequel les grandes villes capitales de vastes entités administratives dispersées sur l'ensemble du territoire sont puissantes (MoriconiEbrard, et al., 1994). L'organisation du territoire ouest-allemand est quasiment inverse du modèle français. Si l'on extrapole à l'échelon national le type rhénan défini par Juillard et Nonn à l'échelle régionale on obtient un type d'organisation défini par Camagni (1992) comme un système réticulé. Dans ce type de configuration interurbaine, les villes sont des nœuds d'interconnexion entre les points distants d'un espace mondial, et la multiplicité des connexions favorise l'accès à l'innovation. Comme dans le type rhénan, les villes fonctionnent en réseau, élargissant ainsi leurs potentialités (Juillard et Nonn, 1976 ; Riquet 1976, 1981). Les recherches urbaines sur ce territoire (Degermann, 1991, Planque, 1976, Riquet (1976, 1979, 1980, 1981), Wild, 1983) ont mis en évidence un net partage interurbain des fonctions économiques rares ou de niveau élevé, entre les villes de niveau international. Les métropoles y ont des spécialisations complémentaires, particulièrement en ce qui concerne les activités tertiaires avancées ou même quaternaires. Un saut existe entre le niveau métropolitain (au sens de Moriconi-Ebrard, 1994) et le reste des villes, il est moins important qu'en France.

Au Royaume-Uni, la forme de la hiérarchie urbaine est proche d'une distribution de Pareto, avec toutefois une légère concavité traduisant l'hypertrophie de la capitale, et une légère faiblesse du niveau des villes intermédiaires de 300000 à un million d'habitants environ (Moriconi-Ebrard et al., 1994). La primauté de la capitale, signalant la présence forte d'un état à l'encadrement territorial dont la centralisation a été renforcée à partir de la fin des années 
1970, est nette, mais quelques très grandes villes au pouvoir fort surnagent et commandent des régions relativement autonomes politiquement (Ecosse, Pays-de-Galles), ou des régions très industrielles (Midlands, Yorkshire, Lancashire) longtemps très dynamiques. Mais, le peu de liens de complémentarité qui lient ces villes, davantage animées par des relations de compétition, apparente davantage la Grande-Bretagne au modèle parisien qu'au modèle rhénan. Ainsi la Grande-Bretagne correspond assez au type parisien avancé décrit à l'échelle régionale par Juillard et Nonn, 1976.

Ainsi, même si l'interaction interurbaine qui s'exprime dans le processus de diffusion des services aux entreprises dépend à la fois de l'accroissement de l'activité des services aux entreprises dans l'espace national, mais aussi de la demande en services aux entreprises dans les villes réceptrices, et par là même de leur spécialisation (le rôle des spécialisations des villes a été envisagée (Michel, 1998), mais n'est pas rapporté ici pour ne pas alourdir le texte ...). Les barrières que constituent les ruptures et discontinuités dans la hiérarchie de taille des villes sont importantes pour expliquer les décalages, entre les trois pays, dans le déroulement de la diffusion. Les barrière ont des effets de freinage dans la propagation du processus. La forme du réseau urbain, et l'articulation des différents niveaux de villes, notamment des métropoles au reste du système modifie le déroulement de la diffusion des services aux entreprises, tout autant que la distribution géographique des villes.

\subsubsection{Effets de barrière et émergence d'un système urbain européen}

Dans le système français, la primatie de la capitale est telle que l'influence qu'elle exerce, notamment pour les fonctions rares est sans partage sur l'ensemble du territoire. Or les services aux entreprises, lors de leur apparition sont une activité rare. Cette prééminence parisienne est telle, qu'au cours de la phase d'émergence, l'ensemble de l'espace national est dépendant de la capitale pour ce type de prestations. Cette dépendance se prolonge au cours de la phase de diffusion précoce, et les autres métropoles économiques sont assez lentes à s'équiper. C'est entre Paris et les villes de rang immédiatement inférieur et pendant la période d'apparition que l'effet de barrière au niveau métropolitain est si fort que l'on peut 1'apparenter à une barrière absorbante (Carte 1 et 2).

Par la suite, lors de la période d'expansion (Carte 3), les services aux entreprises se banalisant cette barrière devient perméable, et une fois les métropoles régionales équipées, le processus de diffusion s'intensifie si bien que les villes de 100000 à un million d'habitants sont rapidement équipées. En effet, une fois le saut majeur dans la hiérarchie urbaine franchi, les différences de taille entre les métropoles régionales et les villes de rang immédiatement inférieur sont faibles, la prééminence qu'elles exercent sur les réseaux régionaux n'est pas suffisamment forte pour inhiber la diffusion au niveau des villes de 100 à 200000 habitants. Ainsi, l'organisation hiérarchique du réseau urbain français favorise une période d'expansion très active, en raison notamment de l'abondance et du continuum statistique des villes de tailles moyennes (de 100 à 500000 habitants). Ces villes restent longtemps à l'écart de l'apparition des services aux entreprises, puis sont ensuite rapidement 'contaminées'. Signalons aussi qu'à partir de la période d'expansion, des barrières perméables apparaissent, bloquant partiellement la diffusion vers les régions anciennement industrialisées. Ces barrières régionales se substituent à la barrière métropolitaine observée à la période d'apparition, mais elles sont bien moins intenses. Lors de la période de condensation (Carte 4), des barrières absorbantes apparaissent dans les vieilles régions industrielles, entre les métropoles d'équilibre et les villes moyennes qui en sont éloignées. La diffusion des services aux entreprises semblent donc pénaliser à terme l'éloignement métropolitain dans les vieilles régions industrielles. 
Dans le système urbain pluricéphale de l'Allemagne de l'Ouest, aucune des huit villes millionnaires n'exerce une influence suffisamment forte sur l'ensemble de l'espace pour être le point prééminent de concentration des services rares pendant la période d'émergence de l'activité (Carte 1 et 2). Même si au cours de cette période le territoire allemand n'est pas unifié, et si des principautés autonomes persistent, Chassaigne (1997) rapporte l'intensification des relations entre les villes des principautés de l'Empire, la mise en place d'un réseau ferroviaire à l'échelle de l'espace allemand, mais qui déjà privilégie les villes d'Allemagne de l'Ouest. Dès 1870, les villes d'Allemagne de l'Ouest constituent un réseau intégré, elles entretiennent entre elles plus de relations qu'elles ne le font avec les autres villes d'Allemagne et d'Europe. De ce fait, au cours de la première période, la diffusion des services aux entreprises est accélérée au niveau actuellement métropolitain. En fait, tout se passe comme si ce niveau correspondait à la seule capitale parisienne. La période d'émergence passée, un effet de seuil apparaît, pendant la diffusion précoce, entre le niveau métropolitain et le reste des villes : une fois que les services aux entreprises se banalisent, et à partir du moment où ils pourraient se répandre plus activement dans l'ensemble des villes, la spécialisation qu'ils expriment reste l'apanage des villes métropolitaines. Le fait que les villes les plus accessibles, les métropoles principales, soient équipées, ralentit et inhibe la diffusion dans l'ensemble du système. Ce seuil se renforce en début de période d'expansion (Carte 3), et on peut alors l'assimiler à une barrière perméable qui isole le niveau métropolitain, du reste du système urbain. A partir du milieu de la période d'expansion, une barrière régionale absorbante, qui oppose vieilles et nouvelles régions industrielles se met en place.

En Grande-Bretagne, lors de la période d'émergence, une barrière perméable existe entre le niveau métropolitain des villes et le reste du système urbain ; elle apparaît entre la dizaine de villes de niveau métropolitain et le reste des villes (Carte 2). C'est au cours de la deuxième phase de la période d'expansion et de développement important des échantillons de sociétés qu'une barrière absorbante apparaît entre Londres et les régions du Sud d'une part et les anciennes régions industrielles des Midlands et du Nord du Royaume-Uni (Carte 3).

Dans le système français, c'est avant la période d'expansion que se manifeste une barrière absorbante très bloquante, alors que dans les systèmes ouest-allemand et britannique il semble qu'une barrière absorbante se mette en place au cours de la période d'expansion, isolant les villes moyennes des vieilles régions industrielles du reste du système urbain. Les barrières métropolitaines peuvent être alternativement bloquantes et perméables ; elles manifestent les sélections spatiales du cycle de l'information. Elles ne disparaissent jamais totalement, même si elles sont perméables : en effet, dans les années 1995, les villes métropolitaines restent le théâtre de la spécialisation dans les services aux entreprises.

Ces résultats, en mettant l'accent sur le rôle de la forme de la hiérarchie urbaine dans le processus de diffusion permettent déjà de dessiner des pistes de recherche sur cette question de la diffusion des innovations économiques. Valoriser la confrontation à un modèle de diffusion épidémiologique en remplaçant l'indicateur de distance par un indicateur d'accessibilité comme cela a été récemment proposé, prendre en compte la position des villes dans des réseaux internationaux, formaliser l'état de la répartition des villes dans l'espace sont autant de pistes de recherche auxquelles on envisage de s'intéresser dans un proche avenir, mais qui n'ont pu être creusée dans le cadre de cette présentation. 


\section{Comportement dynamique des systèmes urbains : quelles modifications dans les}

\section{hiérarchies urbaines ?}

En règle générale, si la diffusion des innovations est souvent vecteur de changement, elle remet rarement en cause la stabilité des systèmes de villes, et bien au contraire, il a souvent été montré que l'organisation hiérarchique des systèmes nationaux est très persistante (Pumain, Saint-Julien, 1978). En effet, ce n'est qu'au cours de la phase d'apparition que les disparités spatiales sont accentuées, modifiant ainsi les hiérarchies en place. On voit ici que la diffusion du cycle de l'information sans remettre en cause les hiérarchies existantes souligne fortement l'avantage métropolitain, et contribue peut-être à l'émergence d'un niveau supérieur de villes. Quelles conséquences cela a-t-il sur la dynamique propre des systèmes urbains nationaux ? Comment les contraintes de localisation des services aux entreprises modifientelles les systèmes urbains nationaux et les discontinuités qu'ils abritent?

\subsection{France : Assez grande mobilité des villes en général, et conservation de l'organisation hiérarchique du système}

L'examen dans les trois systèmes urbains, des modifications de rang (d'après la répartition des sociétés de services aux entreprises) qui se sont produits entre les villes permet de comparer l'intensité des changements qui s'opèrent dans chacun des pays.

Dans le système urbain français, l'avantage initial acquis par les plus grandes villes, et Paris en particulier, ne se démentit jamais, et la position des grandes villes (Paris, Lyon, Lille, Marseille) en tête de l'échantillon n'est jamais réellement remise en cause, tandis que de forts changements peuvent affecter les villes les plus petites de l'échantillon.

On a rangé les villes par ordre croissant selon le nombre d'entreprises qui y sont localisées, entre 1930 et 1994. Les changements de rang sont forts en France et une trentaine de villes rétrograde (17) ou progresse (15). Au delà de ces changements spectaculaires, on peut considérer que les villes montent ou descendent en moyenne de huit rangs en 50 ans. Un certain nombre de villes, surtout des villes de grandes tailles, maintiennent leur position tout au long de la période : ainsi Lille, Lyon, Metz, Nantes, Paris et Rennes (Carte 5). Les changements de position des villes ont lieu principalement pendant les phases d'émergence ou de diffusion précoce des services aux entreprises, tandis que la suite du processus ne fait qu'entériner les modifications apparues antérieurement.

La géographie de l'évolution des villes dans la hiérarchie urbaine de 1930 à 1994 situe donc les changements les plus fortement positifs au nord d'une diagonale Rennes-Nice, notamment dans des villes d'assez grande taille comme Rouen, Saint-Etienne, Reims, Orléans, GrasseCannes-Antibes. Les changements les plus négatifs sont le fait de petites villes de la périphérie du territoire. Les villes qui ont amélioré leur position de quelques rangs sont plutôt situées au sud-ouest d'une diagonale Rouen-Lyon, tandis que les villes qui se sont laissées distancer sont plutôt des villes situées dans la moitié Est de la France (Carte 5).

Au total, dans le système français, les changements de rang des villes sont forts mais un examen des modifications de la répartition relative des sociétés de services aux entreprises montre que la hiérarchie du système est conservée dans son ensemble

\subsection{En Allemagne de l'Ouest : mobilité hiérarchique des villes métropolitaines}

Comme dans le cas français, l'inertie du système est grande au sommet de la hiérarchie, tandis que les changements de rang les plus importants se font essentiellement dans les petites villes. Par rapport à la France, le fait le plus marquant est probablement que l'amplitude des changements de rang est moindre qu'en France. Les villes qui grimpent de manière constante 
dans la hiérarchie urbaine, bien que partant de niveau différents, et à des rythmes divers ont une progression surtout sensible entre 1970 et 1990. Cette progression affecte aussi bien de villes de moins de 500000 habitants (Karlsruhe, Rosenheim, Erlangen, Aix-la-Chapelle) que de grandes villes, mais on notera parmi les grandes la présence de München (Carte 5). Une douzaine de villes baissent dans la hiérarchie urbaine de manière constante : parmi elles, les trois villes principales de Rhénanie du Nord-Westphalie ainsi que Hamburg. Les autres villes globalement changent peu de niveau. En Allemagne de l'Ouest, c'est plus un ensemble de villes du Sud qui se dessine qu'un ensemble métropolitain de villes.

\subsection{En Grande-Bretagne : Mobilité hiérarchique des villes semblables à celle des villes d'Allemagne de l'Ouest}

En Grande-Bretagne, près d'une dizaine de villes gardent leur position : il s'agit des très grandes villes (Londres, Manchester, Birmingham, Liverpool) mais aussi de quelques plus petites villes (Bournemouth, Southend-on-Sea, Norwich, Blackpool, Dundee). Les villes qui perdent des places sont surtout des villes des Midlands, de moins de 500000 habitants, ainsi que quelques villes du sud de Londres (Carte 5). A l'inverse, l'Ecosse, le nord, le nord-est, la zone d'attractivité immédiate de la capitale britannique sont des espaces dans lesquels les villes gagnent des rangs. Parmi les grandes villes à progresser dans cette hiérarchie, on retrouve Newcastle, Glasgow, Edinburgh. Ainsi la "mobilité" descendante ou ascendante des villes sur un axe de l'équipement en services aux entreprises ressemble plus à celle de l'Allemagne de l'Ouest qu'à celle de la France.

Au cours de la période de diffusion massive des sociétés de services aux entreprises, les changements de rang des villes sont d'ampleur différentes selon les trois pays. En France, au cours de la période de diffusion massive, les changements de rang des villes peuvent être de grande amplitude et seules trois villes de têtes conservent leur place (Paris, Lille et Lyon). En Grande-Bretagne, les changements de rang de grande amplitudes (sur un échantillon de villes moins important) sont quasi marginaux, puisqu'ils ne concernent que trois villes; en revanche le nombre de villes qui maintiennent leur position initiale est très important (9), et la très large majorité des villes perd ou gagne entre un et 10 rangs. En Allemagne de l'Ouest, les changements de rang entre les villes s'apparentent davantage à ceux de la Grande-Bretagne, ils sont très faibles dans l'ensemble (entre un et cinq rangs pour la très grande majorité des villes) ; les changements de rang allant au-delà de 10 rangs sont marginaux, puisqu'ils concernent moins d'une dizaine de villes, et que six villes maintiennent exactement leur positions.

Ainsi, on peut associer au réseau macrocéphale français une grande mobilité ascendante ou descendante des villes, particulièrement des villes de moins de 200000 habitants, une mobilité faible des métropoles régionales qui gagnent ou perdent un voire deux rangs pendant l'ensemble de la période, et une stabilité de la position de la capitale d'une part et de deux des capitales régionales les plus importantes Lille et Lyon.

En revanche, dans le réseau ouest-allemand, la mobilité ascendante ou descendante des villes est nettement moins forte dans l'ensemble ; elle affecte aussi des villes de grandes tailles, du niveau supérieur de la hiérarchie urbaine, qui peuvent gagner ou perdre deux, trois, quatre voire six rangs (Essen, Hamburg, Bonn, Berlin, Frankfurt, München), tandis que des villes d'une centaine de milliers d'habitants changent peu de position. Ainsi dans le réseau ouestallemand, la mobilité d'ensemble est faible, mais la mobilité des villes au sein du niveau supérieur de la hiérarchie est forte, tandis que c'est l'inverse en France. 
Dans le réseau urbain britannique, la mobilité ascendante ou descendante des villes sur un axe de l'équipement en services aux entreprises est plutôt faible ; elle s'apparente, par les faibles changements au réseau urbain ouest-allemand, tandis que la stabilité des positions des plus grandes villes la rapproche du réseau français. En France, comme en Allemagne de l'Ouest, les changements de rangs négatifs sont plutôt le fait de villes situées dans les moitiés nord des pays, et les changements de rang positifs dans les moitiés sud.

Au-delà de la "barrière" métropolitaine que l'inégale diffusion des services aux entreprises met en valeur, les comportements et les modes d'assimilation du cycle économique dont font partie les services aux entreprises sont assez différents dans les trois pays. Les dynamiques dont résultent ces configurations sont spécifiques à chacun des systèmes.

En effet, au système primatial français, très hiérarchisé, semblent être associées à la fois une grande mobilité de l'ensemble des villes du système, et une stabilité incontestable de la position des trois plus grandes villes. Mais l'évolution de la répartition relative des services aux entreprises montre que finalement la diffusion des services aux entreprises s'est traduite à la fois par une translation vers le haut de l'ensemble du système, et par une inversion des positions des villes entre le sud et le nord de la France, au profit du sud. D'autre part, ce sont les villes proches des frontières du coeur urbain de l'Europe qui ont vu leurs positions le plus bouleversées. Ainsi, en France, la forme primatiale du réseau semble le garant de la stabilité du niveau métropolitain.

En Allemagne de l'Ouest, il y a inversion des positions des villes au sein du niveau supérieur de la hiérarchie urbaine, au détriment des métropoles du nord et en faveur de celles du sud. La répartition interurbaine des services aux entreprises en 1994, montre qu'un certain nombre de petites villes restent totalement à l'écart du processus de diffusion. En Allemagne de l'Ouest comme en France, la position des villes est plus perturbée à proximité des zones proches du coeur urbain et industriel de l'Europe, et pose la question de l'influence que pourra avoir à la fois la réunification allemande, et l'élargissement de la communauté européenne sur la dynamique d'ensemble du système urbain européen.

\section{Conclusion}

Que conclure de ces résultats en terme de dynamique des systèmes urbains ? Il est tout d'abord important de rappeler que, dans les trois systèmes, pour différentes que soient leurs formes hiérarchiques, la concentration des services aux entreprises se voit globalement renforcée et individualisée au niveau métropolitain. L'état actuel de la diffusion du cycle de l'information, tel qu'il peut être apprécié ici, et la localisation très nettement discriminante des entreprises des échantillons permet de pencher pour le renforcement d'un niveau supérieur de villes dans chaque pays. De nombreuses études laissent penser que ces agglomérations sont davantage de portée européenne que les autres villes des réseaux urbains nationaux (Veltz, 1996, Cattan, et al., 1994), et pourraient constituer la tête multicéphale d'un système urbain européen. Reste à savoir comment s'organisera ce réseau, et comment s'y intégreront les niveaux infra-métropolitains ... Une organisation pluricéphale favorisera-t-elle l'intégration de l'ensemble ou cela sera-t-il plutôt le fait d'une organisation primatiale ?

Il est donc très difficile d'apporter une réponse claire et tranchée qui conclurait à la meilleure absorption des perturbations ou des bénéfices qu'engendre le cycle de l'information dans les trois systèmes urbains. Si en France les rythmes de diffusion évoluent selon une trajectoire qui va d'un rythme très lent à un rythme très rapide de diffusion, pour finalement se stabiliser lorsque toutes les villes du système sont atteintes, en Allemagne de l'Ouest les rythmes sont 
alternativement rapides et lents. Le résultat étant qu'en 1994 une dizaine de villes peu concernées par la diffusion des services aux entreprises sont finalement laissées de côté dans l'ex République Fédérale d'Allemagne. Chacun des trois systèmes enregistre et réagit à la diffusion des services aux entreprises d'une manière spécifique, qu'explique la forme hiérarchique différenciée de chacun.

Force est donc de constater, que malgré un indéniable processus de diffusion, le cycle de l'information renforce l'écart entre des aires métropolitaines comprenant les métropoles et leurs villes satellites (situées dans un rayon qui varie selon les pays mais peut-être évalué au maximum à $200 \mathrm{~km}$ ), et le reste du système urbain. Dans les trois systèmes, des recompositions se dessinent, qui pourraient prolonger la dorsale européenne vers le Sud, et l'affaiblir au Nord, particulièrement au niveau des villes de 100000 à 200000 habitants. De fait, si la stabilité dynamique des systèmes nationaux devait être remise en cause, il semble que ce serait à travers la mise en place d'un système supranational (ici européen) de villes, constitué en priorité, des vastes aires métropolitaines des systèmes nationaux. Les tendances récentes dans la répartition de certaines catégories des services aux entreprises, notamment les services commerciaux et de publicité, à des types de localisations qui associent les capitales et les villes voisines sous leur influence souligne plus encore une logique d'échelle encore supérieure, mondiale, qui met en réseau une catégorie de villes dites globales (Sassen, 1996).

\section{Bibliographie}

Amendola M. and Gaffard J. L. (1988). La dynamique économique de l'innovation, Paris, Economica.

Ascher F. (1995). Métapolis, Paris, Editions Odile Jacob.

Beyers W. B. (1991). «Trends in the producer services in the USA : the last decade ».

Services and metropolitan development, P. W. Daniels (ed), London, Routledge, p. 146-173.

Bourne L. (1978). System of cities, readings on structure, growth and policy, Oxford, Oxford University Press.

Camagni R. (1992). « Organisation économique et réseaux de villes ». Espace et dynamiques territoriales, P. H. Derycke, Economica, pp. 25-52.

Cattan N. et al. (1994). Le système des villes européennes, Paris, Anthropos.

Cattan N., Saint-Julien Th. (1998). « Modèles d'intégration spatiale et réseau des villes en Europe occidentale », Espace géographique, $\mathrm{n}^{\circ} 1$.

Derycke (ed), Economica, p. 25-52.

Castells M. (1989). The informational city, London, Basil Blackwell, $400 \mathrm{p}$.

Daniels P. W. (1985). Service industries, a geographical appraisal, Methuen.

Daniels P. W. (1991), Producer services and the development of the space economy, The changing geography of producer services, P. W. Daniels and F. Moulaert, London, Belhaven Press, pp. 135-151.

Degermann J. (1991). En Europe, des villes en réseau, Datar, Ministère de l'aménagement du territoire, La documerntation française.

Djellal F. and Moulaert F. (1995), "Les firmes de conseil en technologie de l'information.", Revue d'économie régionale et urbaine, 4: 683-704.

Greenfield H. (1966), Manpower and growth of producer services, London, Columbia university press.

Hägerstrand T. (1967). Innovation Diffusion as a spatial process, Chicago, London, University of Chicago Press.

Howells J. R. L. (1984), "The location of research and development : some observations and evidence from Britain.”, Regional studies, 18,(1): 13-29. 
Illeris S. (1991). «Location of services in a service society ». The changing geography of producer services, P. W. Daniels and F. Moulaert, London.

Jouvaud M. (1996). "Vers une typologie des services aux entreprises selon leurs facteurs de localisation.", Revue d'Economie Régionale et Urbaine, t2, p. 597-627.

Juillard E. and Nonn H. (1976). Espaces et régions en Europe occidentale, CNRS, Col des actions thématiques programmées sciences humaines, $\mathrm{n}^{\circ} 10,114 \mathrm{p}$.

Keeble D. and Walker S. (1994). "New firms, small firms and dead firms : spatial patterns and determinants in the UK.". Regional studies, t. 28, n 4 , p. 411-427.

Le Filattre (1964). "La puissance économique des grandes agglomérations françaises". Etudes et Conjoncture, $\mathrm{n}^{\circ} 1$, p. 3-40.

Landes D. S. (1975), The unbound prometheus, Technological change and industrial development in Western Europe from 1750 to present, Cambridge University Press.

Marshall J. N. (1988). Services and uneven development, Oxford University Press.

Michel S., 1998, La diffusion des services aux entreprises dans les systèmes urbains français, ouest-allemand et britannique (1850-1990), Thèse de doctorat, Géographie, Paris I, 450 p. Moriconi-Ebrard F., Pumain D. and V. R. (1994). Evolution récente des trames urbaines en Europe occidentale et orientale, DATAR et le PIR Villes (CNRS).

Moriconi-Ebrard F. (1993). L'urbanisation du monde depuis 1950, Anthropos, Economica. Planque B. (1976). Organisation de l'espace et diffusion du développement (tome 1) : Le contraste France-R.F.A., Aix en Provence, Thèse de doctorat.

Noyelle T. and Peace P. (1991), Information industries : New York's new export base, Services and metropolitan development, P. W. Daniels, London, Routledge.

Pred A. (1975). "Diffusion, organizational spatial structure and city-system development.". Economic Geography, t 51, n³, p. 252-268.

Pumain D. and Saint-Julien T. (1978). Les dimensions du changement urbain, Evolution des structure socio-économique du système urbain français de 1954 à 1975, Paris, CNRS.

Pumain D. and Saint-Julien T., Eds. (1996), Urban networks in Europe, Paris - Londres, John Libbey Eurotext, INED.

Riquet P. (1976), "Secteur tertiaire en Allemagne Fédérale, étude géographique .", Annales de Géographie, 469,:281-332.

Riquet P. (1976), "Secteur tertiaire et métiers tertiaires, approche statistique des activités des services en Allemagne Fédérale.", Annales de Géographie, 467,:1-29.

Riquet P. (1979), Géographie de l'Allemagne fédérale, Paris, Presse Universitaire de France. Riquet P. (1981). "Du Reich Hitlérien aux états actuels, l'armature urbaine de l'espace allemand.", Villes en Parallèle, p. 117-158.

Rogers M. E. (1983). Diffusion of innovations, New-York, Free Press, Macmillan Publishing Co.

Rostow W. W. (1978). The world economy. History and Prospect, Frances Pinter, London. Saint-Julien T. (1982). Croissance industrielle et système urbain, Economica.

Saint-Julien T. (1995). « Diffusion spatiale », Encyclopédie de Géographie, A. Bailly, R.

Ferras and D. Pumain, Paris, Economica, p. 559-581.

Saint-Julien T. and Sabatier L. M. (1996). "La diffusion des services aux entreprises dans le réseau des villes françaises.”, Economie et statistique, T. 294-295, n²4-5, p. 153-163.

Sassen S. (1991). The Global city : New-york, London, Tokyo, Princetown, New-Jersey, Princetown University Press.

Smailes A. E. (1971). "Urban systems.", Transactions of the Institute of British Geographers, t 53, p. 1-14.

Veltz P. (1996), Mondialisation, Villes et Territoires, l'Economie d'Archipel, Paris, Presses Universitaires de France.

Wild T., Ed. (1983). Urban and rural change in West Germany, Croom Helm Ltd. 
Tableau 1. : nombre de sociétés, nombre de villes touchées (dans lesquelles on trouve des sociétés de services aux entreprises (10 en France et en Allemagne, 5 en Grande-Bretagne en raison de la moindre taille de l'échantillon)

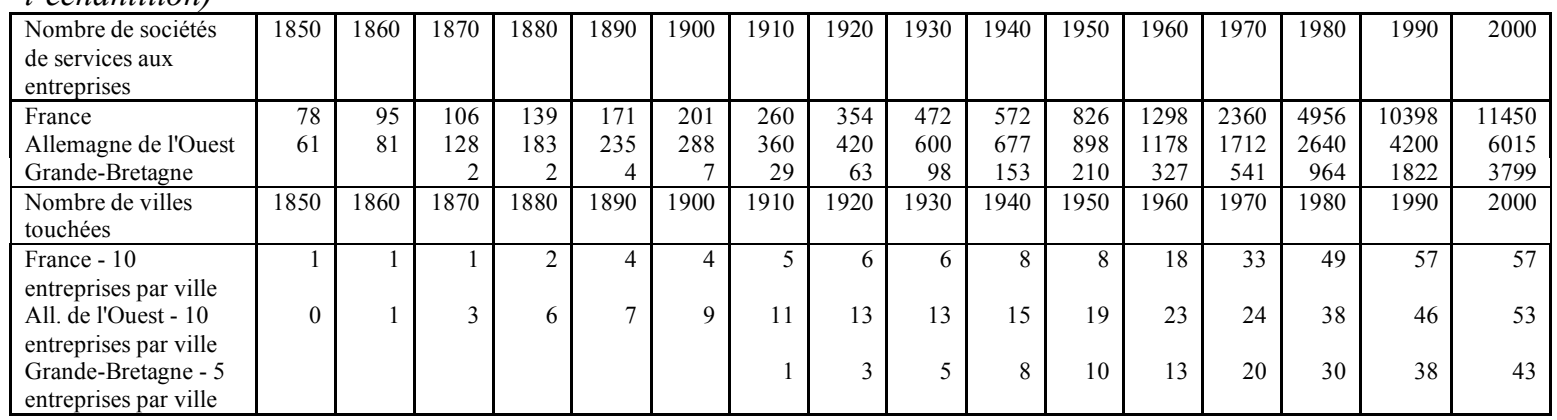

Tableau $2:$ Les 3 périodes de la diffusion des services aux entreprises

\begin{tabular}{|c|c|c|c|c|}
\hline Période & Apparition & & Expansion & Condensation \\
\hline Phase & Emergence & Diffusion précoce & & \\
\hline $\begin{array}{l}\text { France } \\
\text { France - Nombre de villes touchées } \\
\text { en plus au terme de chaque période }\end{array}$ & $\begin{array}{l}1850-1930[ \\
6\end{array}$ & $\begin{array}{l}1930-1950[ \\
2\end{array}$ & $\begin{array}{l}1950-1990[ \\
51\end{array}$ & $\begin{array}{l}1990-1994 \\
0\end{array}$ \\
\hline $\begin{array}{l}\text { Allemagne de l'Ouest } \\
\text { All. de l'Ouest - Nombre de villes } \\
\text { touchées en plus au terme de chaque } \\
\text { période }\end{array}$ & $\begin{array}{l}1850-1930[ \\
13\end{array}$ & $\begin{array}{l}1930-1970[ \\
28\end{array}$ & $\begin{array}{l}1970-1990[ \\
33\end{array}$ & $\begin{array}{l}1990-1994 \\
7\end{array}$ \\
\hline $\begin{array}{l}\text { Grande-Bretagne } \\
\text { G.B. - Nombre de villes touchées en } \\
\text { plus au terme de chaque période }\end{array}$ & & $\begin{array}{l}1910-1960[ \\
13\end{array}$ & $\begin{array}{l}1960-1994 \\
25\end{array}$ & \\
\hline
\end{tabular}


Figure 1. : Taux annuel d'accroissement des sociétés de services aux entreprises (le nombre de sociétés de services aux entreprises pour une décennie / nombre de sociétés à la décennie précédente)

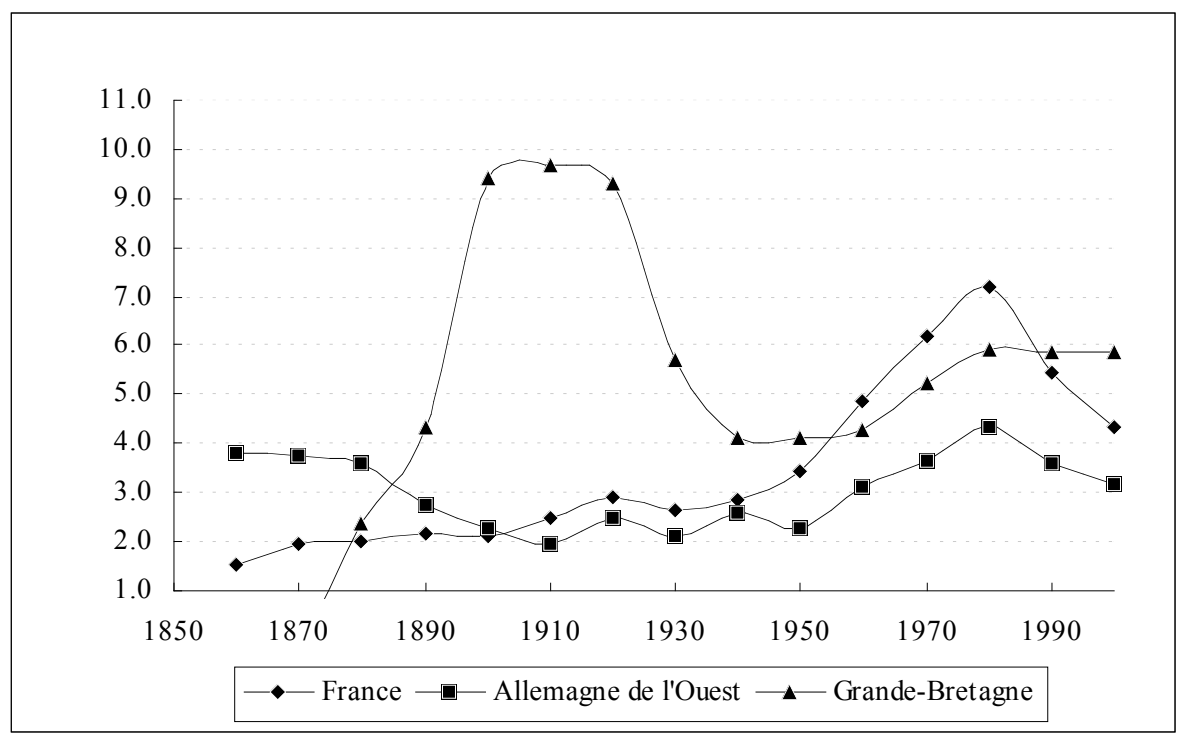

Figure 2. : Taux annuel de diffusion des sociétés de services aux entreprises dans les villes (le nombre de villes touchées à chaque période / nombre de villes touchées à la période précédent)

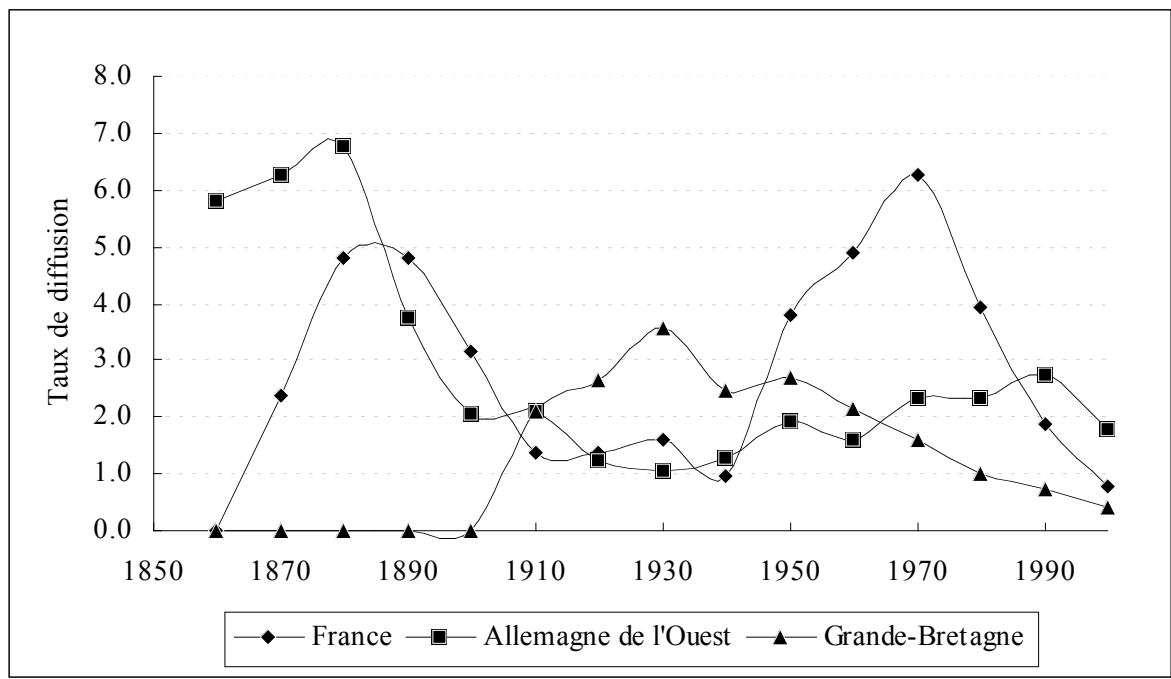


Figure 3. : Le nombre de villes dans lesquelles on trouve des sociétés de services aux entreprises (au moins 10 par ville en France, Allemagne de l'Ouest, au moins 5 GrandeBretagne ; le seuil est inférieur en Grande-Bretagne en raison du sous-enregistrement des sociétés)

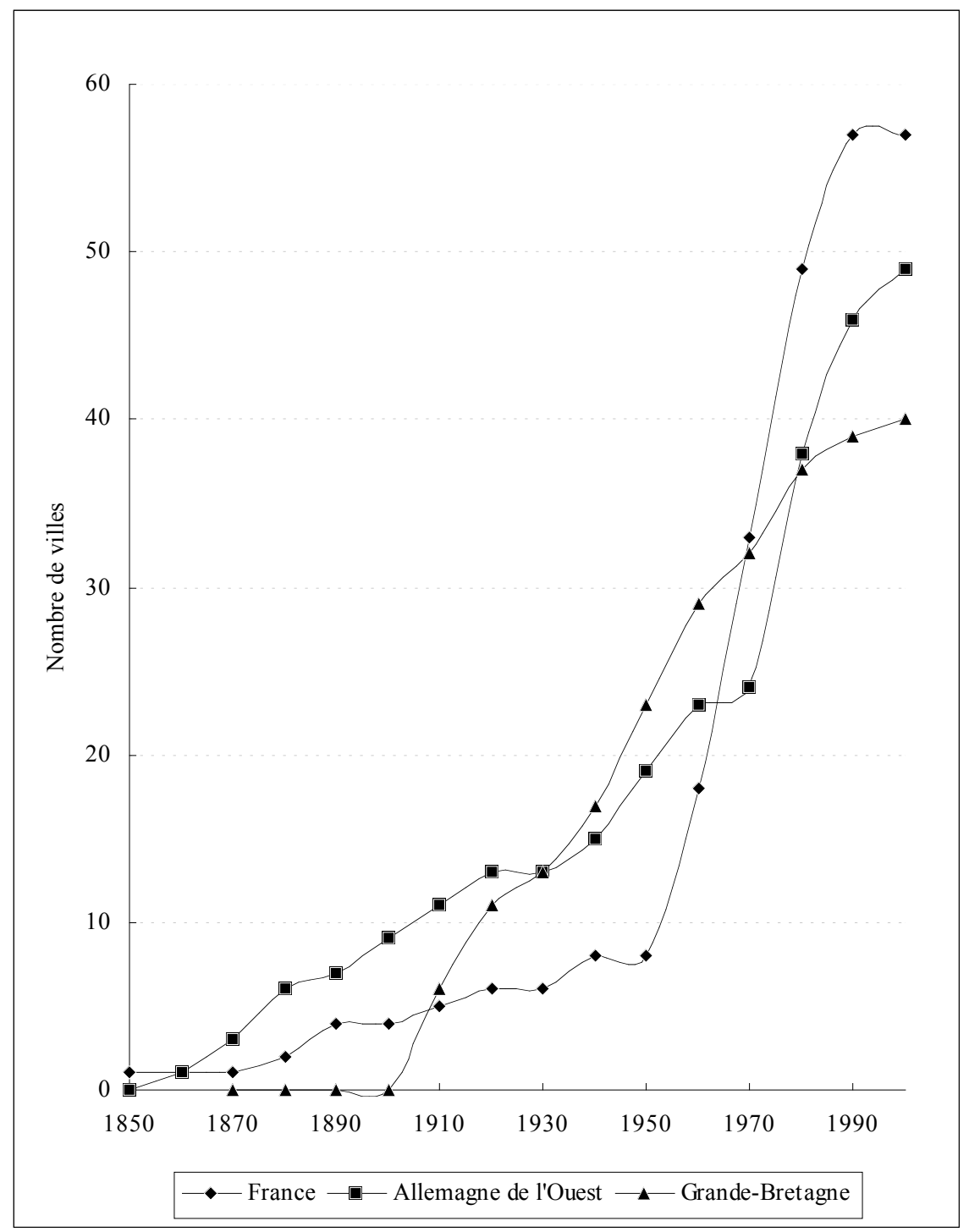


Figure 4.17.a : France : Les changements de rang dans les villes entre 1930 et 1994 d'après le nombre de sociétés de services aux entreprises

P1990 : Population en 1990 (en milliers d'habitants)

Rg94-Rg30 : Les baisses ou les hausses de rang entre 1930 et 1994

Figure 4.17. b : All. de l'Ouest : Les changements de rang des villes entre 1930 et 1994

d'après le nombre de sociétés de services aux entreprises 
Sum-e1990- : Population en 1990 (en milliers d'habitants)- Rg30-

Rg94 : Les baisses ou les hausses de rang entre 1930 et 1994

Source : géopolise - Kompass 
Figure 4.17. c : Grande- Bretagne : Les changements de rang des villes entre 1960 et 1996 d'après le nombre de sociétés de services aux entreprises

P1981 - : Population en 1981 (en milliers d'habitants)

Rg60-Rg96 : Les baisses ou les hausses de rang entre 1960 et 1996

Source : géopolise - Kompass 